\title{
Spousal Concordance regarding Lifestyle Factors and Chronic Diseases among Couples Visiting Primary Care Providers in Korea
}

\author{
Seung Yup Jun, Mina Kang, Seo Young Kang, Jung Ah Lee*, Young Sik Kim \\ Department of Family Medicine, Asan Medical Center, University of Ulsan College of Medicine, Seoul, Korea
}

\begin{abstract}
Background: Few studies have been conducted in Korea on concordance regarding chronic diseases and lifestyle factors among couples. We, therefore, evaluated spousal concordance regarding lifestyle factors and chronic diseases among Korean couples.

Methods: A total 1,040 participants (520 couples) who visited family physicians were recruited from 22 hospitals. All participants were aged $\geq 40$ years. Participants completed questionnaires on smoking, drinking, physical activity, and irregular eating habits, including skipping breakfast. We estimated the spousal concordance regarding lifestyle factors and chronic diseases using McNemar tests and logistic regression analysis.

Results: The concordance rate was high among couples who shared unhealthy behaviors, such as low physical activity, irregular diet, and skipping breakfast $(\mathrm{P}<0.05)$. When cardiovascular risk factors such as overweight (odds ratio [OR], 1.73; 95\% confidence interval [CI], 1.14-2.63), hypertension (OR, 1.88; 95\% CI, 1.23-2.86), or hyperlipidemia (OR, 2.41; 95\% CI, 1.60-3.64) were prevalent among husbands, the odds that their wives also had the same risk factors at the same time was significantly high. The odds of being depressed when the spouse was depressed were also significantly high in both men (OR, 5.54; 95\% CI, 2.19-13.96) and women (OR, 4.52; 95\% CI, 1.77-11.53). Conclusion: There was a high level of concordance regarding lifestyle factors among couples, which could lead to an increase in the prevalence of chronic diseases among couples. In addition, if a spouse has depression, the odds of their partner also having depression was high.
\end{abstract}

Keywords: Spouses; Couple; Chronic Disease; Risk Factors; Concordance; Life Style

Received: July 12, 2018, Revised: October 29, 2018, Accepted: November 19, 2018

*Corresponding Author: Jung Ah Lee https://orcid.org/0000-0003-3125-7805

Tel: +82-2-3010-3810, Fax: +82-2-3010-3815, E-mail: ljunga00@naver.com 


\section{INTRODUCTION}

Marriage is a core relationship between an adult couple, which can deeply affect both physical ${ }^{1-3)}$ and mental health. ${ }^{4)}$ Married couples usually spend a large amount of time together, sharing both their environment and lifestyle factors. ${ }^{5)}$ Because spouses are not biologically related, we can speculate that shared lifestyle factors can impact their health outcomes. ${ }^{6}$ Thus, it is important to determine the relationship between coincident health behaviors and chronic diseases among couples to better understand the etiology and management of chronic diseases.

Previous studies have shown that couples are more likely to have similar chronic illnesses. ${ }^{7}$ A previous cross-sectional study revealed that individuals living with their spouses who had asthma, depression, hypertension, hyperlipidemia, or gastric ulcers were more likely to have the same diseases, as opposed to individuals living with spouses who did not have those diseases. ${ }^{8)}$ In a cohort study involving 4,500 couples, an association was observed between hypertensive status in spouses and the likelihood of receiving treatment, after adjusting for shared environmental factors. ${ }^{9)}$ Additionally, spousal concordance for elevated blood glucose was identified in a study conducted among an elderly population. ${ }^{10)}$

A recent study has reported concordance between physical activity and its changes among spouses. ${ }^{11)}$ Another study has reported that obesity among couples can be affected by social influences such as a partner's health behaviors, including exercise and dietary patterns. ${ }^{12)}$

In Korea, a previous cross-sectional study involving 377 couples showed significant spousal concordance for body mass index (BMI), blood pressure, cholesterol, and blood sugar. ${ }^{13)}$ However, few studies have investigated the spousal concordance regarding lifestyle factors associated with cardiovascular health risks among married couples in Korea. The purpose of this study was to investigate the relationship between health status, such as being overweight or having hypertension, diabetes mellitus, hyperlipidemia, or depression, and common shared lifestyle factors such as drinking, smoking, exercise, and dietary patterns, among couples who visited the family medicine department of 22 hospitals in Korea. In addition, we investigated the concordance of depression among Korean couples.

\section{METHODS}

\section{Study Participants}

This study was based on data collected for the Family Cohort Study in Primary Care. The protocol of this study and written informed consent form were approved by the institutional review board of Asan Medical Center (IRB approval no., 2010-0370). Participants read and signed the informed consent form prior to the start of the study. Cohort data were collected for heterosexual couples, including individuals who were married, cohabitating, separated, and divorced. The recruited participants (couples) were individuals aged between 30 and 80 years who had visited the department of family medicine at hospitals nationwide for the treatment of chronic diseases such as hypertension, type 2 diabetes, and dyslipidemia, or for a periodic health checkup, between April 2009 and June 2011 in any of the 22 university-affiliated hospitals of Korea. A total of 1,040 participants (520 couples) were enrolled.

\section{Variables and Measurement}

Demographic characteristics and health behaviors were prospectively

Table 1. Basic characteristics of study participants

\begin{tabular}{|c|c|c|c|}
\hline Characteristic & $\begin{array}{l}\text { Husbands } \\
(\mathrm{N}=520)\end{array}$ & $\begin{array}{l}\text { Wives } \\
(\mathrm{N}=520)\end{array}$ & P-value \\
\hline Age (y) & & & $<0.001$ \\
\hline$<50$ & $91(17.6)$ & $119(23.0)$ & \\
\hline $50-59$ & $141(27.2)$ & $199(38.4)$ & \\
\hline $60-69$ & $204(39.4)$ & $174(33.6)$ & \\
\hline$\geq 70$ & $82(15.8)$ & $26(5.0)$ & \\
\hline Education (y) & & & $<0.001$ \\
\hline$>12$ & $292(56.7)$ & $188(36.5)$ & \\
\hline 12 & $147(28.5)$ & $186(36.1)$ & \\
\hline$<12$ & $76(14.8)$ & $141(27.4)$ & \\
\hline Monthly income $(10,000 \mathrm{KRW} / \mathrm{mo})$ & & & 0.633 \\
\hline$\geq 600$ & $151(29.8)$ & $131(26.6)$ & \\
\hline $400-599$ & $109(21.5)$ & $110(22.3)$ & \\
\hline 200-399 & $157(31.0)$ & $154(31.2)$ & \\
\hline$<200$ & $89(17.6)$ & $98(19.9)$ & \\
\hline Body mass index $\left(\mathrm{kg} / \mathrm{m}^{2}\right)$ & & & $<0.001$ \\
\hline$<18.5$ & $3(0.7)$ & $9(2.0)$ & \\
\hline $18.5-22.9$ & $102(22.9)$ & $160(35.9)$ & \\
\hline $23.0-24.9$ & $124(27.9)$ & $130(29.1)$ & \\
\hline$\geq 25.0$ & $216(48.5)$ & $147(33.0)$ & \\
\hline Current smoker & & & $<0.001$ \\
\hline No & $409(78.7)$ & $514(98.8)$ & \\
\hline Yes & $111(21.3)$ & $6(1.2)$ & \\
\hline Risky alcohol use & & & $<0.001$ \\
\hline No & $157(39.9)$ & $169(59.1)$ & \\
\hline Yes & $236(60.1)$ & $117(40.9)$ & \\
\hline Low physical activity & & & 0.014 \\
\hline No & $169(41.5)$ & $184(50.4)$ & \\
\hline Yes & $238(58.5)$ & $181(49.6)$ & \\
\hline Irregular diet & & & 0.016 \\
\hline No & 463 (89.6) & $436(84.3)$ & \\
\hline Yes & $54(10.4)$ & $81(15.7)$ & \\
\hline Breakfast skipping & & & 0.016 \\
\hline No & $450(87.2)$ & $421(81.6)$ & \\
\hline Yes & $66(12.8)$ & 95 (18.4) & \\
\hline Hypertension & & & $<0.001$ \\
\hline No & $259(49.8)$ & $322(61.9)$ & \\
\hline Yes & $244(46.9)$ & $180(34.6)$ & \\
\hline Diabetes mellitus & & & $<0.001$ \\
\hline No & 362 (69.6) & $437(84.0)$ & \\
\hline Yes & $141(27.1)$ & 64 (12.3) & \\
\hline Hyperlipidemia & & & 0.076 \\
\hline No & 301 (57.9) & $335(64.4)$ & \\
\hline Yes & $202(38.8)$ & $167(32.1)$ & \\
\hline Depressive mood & & & 0.001 \\
\hline No & $434(90.2)$ & $390(82.8)$ & \\
\hline Yes & $47(9.8)$ & $81(17.2)$ & \\
\hline
\end{tabular}

Values are presented as mean \pm standard deviation or number (\%). 
collected using a standardized questionnaire, which included questions regarding educational status, monthly income, smoking status, alcohol intake, physical activity, and dietary pattern. An interviewer or primary care physician collected individual medical histories in person and recorded any prior history of hypertension, diabetes, and hyperlipidemia.

Educational level was categorized into three groups: $<12$ years, 12 years, and $>12$ years. Monthly income was assessed using total household income, queried with a single question. Monthly income was divided into four categories: $<2.00$ million won (1,715 US dollar [USD]), 2.00-3.99 million won (1,715-3,430 USD), 4.00-5.99 million won (3,430-5,145 USD), and $\geq 6.00$ million won (5,145 USD). Smoking status was classified as current smoker, ex-smoker, or never-smoker based on the definition of smokers established by the US Centers for Disease Control and Prevention. ${ }^{14)}$ Never-smokers were participants who smoked fewer than 100 cigarettes in their entire lifetime; participants who had smoked more than 100 cigarettes during their lifetime but were not smoking at the time of this study were classified as exsmokers; and current smokers were participants who smoked more than 100 cigarettes during their lifetime and currently continued to smoke cigarettes. The Alcohol Use Disorders Identification Test-Korean version (AUDIT-K) was used to assess risky alcohol consumption, defined as a total AUDIT-K score of $\geq 8$. ${ }^{15)}$ Physical activity was estimated using the International Physical Activity Questionnaire Short-Form, which evaluates the intensity and duration of physical activity performed during the previous 7 days. ${ }^{16)}$ Physical activity was classified as low, moderate, and high. Low activity level was defined as attainment of $<1,500$ metabolic equivalent min/wk. Dietary patterns were assessed with a question about the regularity of meal intake, which was divided into always regular, usually regular, or irregular, as well as a question about the frequency of breakfast intake.

The presence of hypertension, diabetes, or dyslipidemia was defined either using participants' self-report of a disease or from the medical records indicating the use of antihypertensive medications, oral hypoglycemic agents, insulin, or lipid-lowering agents. Depressive mood was evaluated using a Korean-type Center for Epidemiologic Studies Depression Scale, with depressive mood assessed as a score of $\geq 21$ points. ${ }^{17)}$

Height and body weight were measured to the nearest $0.1 \mathrm{~cm}$ and 0.1 $\mathrm{kg}$, respectively, by trained interviewers. BMI was calculated as (weight in $\mathrm{kg}$ ) $/(\text { height in } \mathrm{m})^{2}$ and was categorized into four groups: $<18.5 \mathrm{~kg} / \mathrm{m}^{2}$, $18.5-22.9 \mathrm{~kg} / \mathrm{m}^{2}, 23.0-24.9 \mathrm{~kg} / \mathrm{m}^{2}$, and $\geq 25.0 \mathrm{~kg} / \mathrm{m}^{2}$. BMI $\geq 25.0 \mathrm{~kg} / \mathrm{m}^{2}$ was defined as overweight, according to 2000 World Health Organization criteria. ${ }^{18)}$

\section{Statistical Analysis}

The chi-square test was used to compare the characteristics of husbands and wives. To evaluate spousal concordance, McNemar tests were used in paired couples. Binary logistic regression analysis was used to estimate the odds ratios (ORs) and 95\% confidence intervals (CIs) for lifestyle factors and chronic diseases among spouses after grouping husbands and wives. In addition, potential confounders such as age, education, and income were adjusted in the logistic regression analysis. All statistical analyses were performed using IBM SPSS ver. 21.0 (IBM Corp., Armonk, NY, USA). A P-value of $<0.05$ was considered to indicate statistical significance.

\section{RESULTS}

\section{Basic Characteristics of Study Participants}

Table 1 shows the basic characteristics of study participants, grouped into husbands and wives. Most participants (69\%) were aged between 50 and 70 years. Approximately $56.7 \%$ of husbands and $36.5 \%$ of wives had completed $>12$ years of education. More husbands (21.3\%) than wives $(1.2 \%)$ were current smokers. A total $60.1 \%$ of husbands and $40.9 \%$ of wives engaged in risky alcohol consumption. About half of both husbands and wives (58.5\% and $49.6 \%$ ) had low physical activity. The frequency of irregular meal intake and skipping breakfast was higher among wives than among husbands, with $15.7 \%$ of wives reporting irregular meal intake and $18.4 \%$ reporting skipping breakfast;

Table 2. Cross-sectional analysis of spousal concordance for chronic disease and lifestyle factors

\begin{tabular}{|c|c|c|c|c|c|c|}
\hline \multirow{2}{*}{ Characteristic } & \multirow{2}{*}{ No. of pairs } & \multicolumn{4}{|c|}{ Paired data } & \multirow{2}{*}{ P-value* } \\
\hline & & $H+M+(\%)$ & $\mathrm{H}+\mathrm{W}-(\%)$ & $H-N+(\%)$ & H-M- (\%) & \\
\hline Total & 520 & & & & & \\
\hline Current smoking & 520 & $3(0.6)$ & $108(20.8)$ & $3(0.6)$ & $406(78.1)$ & $<0.001$ \\
\hline Risky alcohol use & 240 & $69(28.8)$ & 78 (32.5) & 32 (13.3) & $61(25.4)$ & $<0.001$ \\
\hline Low physical activity & 306 & $81(26.5)$ & $47(15.4)$ & 73 (23.9) & $105(34.3)$ & 0.022 \\
\hline Irregular diet & 517 & $24(4.6)$ & $30(5.8)$ & $57(11.0)$ & 406 (78.5) & 0.005 \\
\hline Breakfast skipping & 515 & $38(7.4)$ & $28(5.4)$ & $57(11.1)$ & $392(76.1)$ & 0.002 \\
\hline Overweight & 442 & $81(18.3)$ & $128(29.0)$ & $65(14.7)$ & $168(38.0)$ & $<0.001$ \\
\hline Hypertension & 497 & $118(23.7)$ & $123(24.7)$ & $62(12.5)$ & $194(39.0)$ & $<0.001$ \\
\hline Diabetes & 496 & $28(5.6)$ & $111(22.4)$ & $35(7.1)$ & $322(64.9)$ & $<0.001$ \\
\hline Dyslipidemia & 497 & $97(19.5)$ & $103(20.7)$ & $68(13.7)$ & $229(46.1)$ & 0.009 \\
\hline Depression & 447 & $9(2.0)$ & $17(3.8)$ & $36(8.1)$ & $385(86.1)$ & 0.013 \\
\hline
\end{tabular}


only $10.4 \%$ of husbands reported irregular meal intake and $12.8 \%$ reported skipping breakfast. In terms of comorbidities, hypertension was the most common disease among couples (46.9\% versus $34.6 \%$ ), and the prevalence of diabetes was 2 times higher among husbands than among wives (27.1\% versus $12.3 \%)$. Conversely, depressive mood was higher in wives (17.2\%) than in husbands (9.8\%).

\section{Spousal Concordance regarding Lifestyle Factors and Chronic Diseases}

Table 2 shows spousal concordance regarding lifestyle factors and chronic diseases. Among husband and wife pairs, we analyzed the prevalence of both couples having current smoker status (0.6\%), risky alcohol consumption (28.8\%), low physical activity (26.5\%), irregular diet (4.6\%), and skipping breakfast (7.4\%). In terms of chronic diseases, the prevalence of excessive weight, hypertension, diabetes, and dyslipidemia among both partners in a couple was $18.3 \%, 23.7 \%, 5.6 \%$, and $19.5 \%$, respectively. Moreover, spousal concordance regarding depressive mood was $2 \%$.

\section{Logistic Regression Analysis for Individual Odds Ratio according to Lifestyle Factors and Chronic Diseases among Spouses}

Table 3 shows the results of logistic regression analysis for individual ORs according to lifestyle factors and chronic disease status of spouses. We calculated age-adjusted ORs and multivariate ORs, adjusted for age, years of education, and income level. In terms of lifestyle factors, low physical activity, irregular diet, and skipping breakfast showed significant spousal concordance. When husbands had low physical activity, the OR of wives for low physical activity was 2.47 (OR, 2.47; 95\% CI, 1.54-3.96). When husbands had irregular meal intake, the odds of their wives having irregular meal intake were 4 times higher than those of wives whose husbands ate regularly (OR, 4.30; 95\% CI, 3.75-12.48). Moreover, when husbands skipped breakfast, the spousal concordance was high (OR, 6.84; 95\% CI, 3.42-11.45). Spousal concordance was also present in couples who were overweight or who had hypertension, diabetes, dyslipidemia, or depressive mood. Participants living with overweight spouses had higher odds of being overweight (OR,

Table 3. Logistic regression analysis for individual odds ratios according to spouse's lifestyle factors and chronic diseases

\begin{tabular}{|c|c|c|c|c|}
\hline \multirow{2}{*}{ Spouse's status } & \multicolumn{2}{|c|}{ Husbands } & \multicolumn{2}{|c|}{ Wives } \\
\hline & Age-adjusted & Multivariate* & Age-adjusted & Multivariate* \\
\hline \multicolumn{5}{|l|}{ Current smoking } \\
\hline No (ref) & 1 & 1 & 1 & 1 \\
\hline Yes & $2.81(0.55-14.28)$ & $1.96(0.37-10.40)$ & $2.97(0.58-15.14)$ & $5.29(0.78-36.04)$ \\
\hline \multicolumn{5}{|l|}{ Risky alcohol use } \\
\hline No (ref) & 1 & 1 & 1 & 1 \\
\hline Yes & $1.53(0.88-2.68)$ & $1.48(0.84-2.61)$ & $1.63(0.93-2.86)$ & $1.59(0.89-2.84)$ \\
\hline \multicolumn{5}{|l|}{ Low physical activity } \\
\hline No (ref) & 1 & 1 & 1 & 1 \\
\hline Yes & $2.46(1.54-3.94)$ & $2.47(1.54-3.96)$ & $2.49(1.56-3.98)$ & 2.39 (1.49-3.85) \\
\hline \multicolumn{5}{|l|}{ Irregular diet } \\
\hline No (ref) & 1 & 1 & 1 & 1 \\
\hline Yes & $5.20(2.82-9.55)$ & $4.30(2.26-8.20)$ & $5.02(2.71-9.29)$ & $4.08(2.12-7.86)$ \\
\hline \multicolumn{5}{|l|}{ Breakfast skipping } \\
\hline No (ref) & 1 & 1 & 1 & 1 \\
\hline Yes & $6.64(3.67-12.01)$ & $6.84(3.75-12.48)$ & $6.25(3.42-11.45)$ & $5.14(3.30-11.41)$ \\
\hline \multicolumn{5}{|l|}{ Overweight } \\
\hline No (ref) & 1 & 1 & 1 & 1 \\
\hline Yes & $1.62(1.09-2.43)$ & $1.73(1.14-2.63)$ & $1.60(1.07-2.40)$ & 1.72 (1.13-2.62) \\
\hline \multicolumn{5}{|l|}{ Hypertension } \\
\hline No (ref) & 1 & 1 & 1 & 1 \\
\hline Yes & $1.88(1.24-2.84)$ & $1.88(1.23-2.86)$ & $1.83(1.21-2.77)$ & $1.93(1.26-2.97)$ \\
\hline \multicolumn{5}{|l|}{ Diabetes } \\
\hline No (ref) & 1 & 1 & 1 & 1 \\
\hline Yes & $1.82(1.04-3.19)$ & $1.64(0.92-3.92)$ & $1.90(1.09-3.31)$ & $1.70(0.96-3.00)$ \\
\hline \multicolumn{5}{|l|}{ Dyslipidemia } \\
\hline No (ref) & 1 & 1 & 1 & 1 \\
\hline Yes & $2.46(1.64-3.69)$ & $2.41(1.60-3.64)$ & $2.41(1.60-3.62)$ & $2.53(1.66-3.86)$ \\
\hline \multicolumn{5}{|l|}{ Depression } \\
\hline № (ref) & 1 & 1 & 1 & 1 \\
\hline Yes & $5.69(2.36-13.70)$ & $5.54(2.19-13.96)$ & $5.66(2.35-13.63)$ & $4.52(1.77-11.53)$ \\
\hline
\end{tabular}

Values are presented as odds ratio (95\% confidence interval) for having identical lifestyle factors or chronic diseases among spouses. Ref, reference.

${ }^{*}$ Adjusted for age, education, and income. 
1.73; 95\% CI, 1.14-2.63 for husbands; OR, 1.72; 95\% CI, 1.13-2.62 for wives). Furthermore, husbands with wives who had hypertension, dyslipidemia, or depressive mood had significantly higher odds of having hypertension (OR, 1.88; 95\% CI, 1.23-2.86), dyslipidemia (OR, 2.41; 95\% CI, 1.60-3.64), or depression (OR, 5.54; 95\% CI, 2.19-13.96). Similar results were found among wives with husbands who had hypertension (OR, 1.93; 95\% CI, 1.26-2.97), dyslipidemia (OR, 2.53; 95\% CI, 1.66-3.86), and depressive mood (OR, 4.52; 95\% CI, 1.77-11.53).

\section{DISCUSSION}

In our study, we investigated the spousal concordance regarding cardiovascular risk factors among Korean couples. The rate of spousal concordance was high with respect to sharing unhealthy behaviors, including low physical activity levels, irregular meal intake, and skipping breakfast. In addition, the odds of being overweight as well as for having high blood pressure or dyslipidemia were twice as high among individuals whose spouses had these diseases as among individuals whose spouses did not have these diseases. The odds of having depressive mood were especially associated with an individual's spouse also having depressive mood, found to be 5.5 times higher in husbands and 4.5 times higher in wives.

Low physical activity and sedentary lifestyle are well-known risk factors for cardiovascular diseases. A meta-analysis reported spousal concordance regarding cardiovascular risk factors including hypertension, smoking, diabetes, and obesity. ${ }^{2)}$ Regarding the sharing of unhealthy behaviors among couples, the results of this study are consistent with those of previous studies. ${ }^{5,11)}$ A study that evaluated the familial aggregation of physical activity also found that spousal correlation regarding physical activity was occasionally higher than the correlation between same-sex siblings. ${ }^{19)}$ These results suggest that physical activity could be affected by both a shared environment and genetic factors. With regard to eating habits, $7.4 \%$ of the couples in our study reported that they skipped breakfast whereas $76.1 \%$ reported regularly eating breakfast. Thus, the discordance of skipping breakfast was only $16.5 \%$, which was the lowest among the lifestyle factors investigated in our study. Similar results were obtained with regard to concordance for irregular eating habits. We suspect that couples are likely to have similar healthy habits owing to the sharing of health information, such as the positive influence of eating breakfast on health.

According to a systematic review, many previous studies have found spousal concordance for weight, obesity, blood pressure, and cholesterol level. ${ }^{20,21)}$ In one Korean study, the researchers found spousal concordance for metabolic syndrome when multiple diseases such as hypertension, hyperlipidemia, obesity, and cardiovascular atherosclerosis were observed in one individual. ${ }^{22)}$ A previous study among Korean family members demonstrated that spousal concordance for cardiovascular risk factors increased with the partner's age. ${ }^{23)}$ Similar to these results, our study revealed that the prevalence of excessive body weight, hypertension, or dyslipidemia among couples was associated with chronic diseases among spouses, after adjusting for age, educa- tion, and income. Spousal concordance regarding such diseases was present independently of age and socioeconomic status because the results remained consistent with those before adjusting for the above factors.

In our study, there was also a high concordance for depression between individuals and their spouses. People with spouses who are experiencing depressive mood had 4-5 times higher odds of having a depressed mood themselves. These findings are supported by previous studies revealing that when one spouse is depressed, the other spouse tended to have a similarly depressed mood. ${ }^{24)}$ In many cases, we can speculate that concordance for depressive symptoms is more likely after a major life event for one of the partners, such as a cancer diagnosis. ${ }^{25)}$ Although we did not investigate the reasons for depressive mood, we suspect that these can be attributed to a couple's life experiences. One study revealed concordance in the experience of depressive episodes among couples in a community cohort, regardless of the associated symptomatology; it also suggested a weak concordance for the lifetime prevalence of major depression. ${ }^{26)}$

Our study has several limitations. Because our study had a crosssectional design, it was difficult to evaluate the effect of spousal concordance on the actual occurrence of a cardiovascular disease. Additionally, because we did not evaluate the marriage duration among our study couples, we could not determine the long-term and shortterm effects of marriage on chronic diseases and lifestyle factors. Moreover, as participants were recruited mainly from the department of family medicine in general hospitals, we cannot directly apply our findings to all primary care patients in Korea. However, to our knowledge, our study is the first to recruit couples visiting primary care clinics in Korea and to evaluate various lifestyle factors and common chronic conditions associated with cardiovascular diseases. Ours is also one of the few studies to evaluate depressive mood among couples in Korea.

Despite these limitations, our study presents important findings regarding spousal concordance regarding chronic diseases and lifestyle factors among Korean couples, which has rarely been investigated in Asian countries. In addition, several Korean studies have previously focused on cardiovascular risk factors among couples but have not investigated their correlation with emotional status. Moreover, our study included a large sample of couples who visited the department of family medicine at hospitals nationwide.

In conclusion, there was a high concordance with respect to lifestyle factors and chronic diseases between married couples in our study, which might in turn lead to an increased prevalence of cardiovascular diseases among those couples. In addition, we found that if one spouse had depression, the likelihood of the partner feeling depressed was also very high. To provide high-quality primary care to couples, clinicians should keep in mind the high spousal concordance regarding risk factors and diseases. 


\section{CONFLICT OF INTEREST}

No potential conflict of interest relevant to this article was reported.

\section{ORCID}

Seung Yup Jun: https://orcid.org/0000-0003-3827-136X

Mina Kang: https://orcid.org/0000-0002-9614-9664

Seo Young Kang: https://orcid.org/0000-0002-7177-7816

Jung Ah Lee: https://orcid.org/0000-0003-3125-7805

Young Sik Kim: https://orcid.org/0000-0002-1786-4273

\section{REFERENCES}

1. Waite LJ. Does marriage matter? Demography 1995;32:483-507.

2. Di Castelnuovo A, Quacquaruccio G, Donati MB, de Gaetano G, Iacoviello L. Spousal concordance for major coronary risk factors: a systematic review and meta-analysis. Am J Epidemiol 2009;169:1-8.

3. Schwandt HM, Coresh J, Hindin MJ. Marital status, hypertension, coronary heart disease, diabetes, and death among African American women and men: incidence and prevalence in the Atherosclerosis Risk in Communities (ARIC) study participants. J Fam Issues 2010;31: 1211-29.

4. Townsend AL, Miller B, Guo S. Depressive symptomatology in middleaged and older married couples: a dyadic analysis. J Gerontol B Psychol Sci Soc Sci 2001;56:S352-64.

5. Kolonel LN, Lee J. Husband-wife correspondence in smoking, drinking, and dietary habits. Am J Clin Nutr 1981;34:99-104.

6. Pyke SD, Wood DA, Kinmonth AL, Thompson SG. Change in coronary risk and coronary risk factor levels in couples following lifestyle intervention: the British Family Heart Study. Arch Fam Med 1997;6:354-60.

7. Inoue K, Sawada T, Suge H, Nao Y, Igarashi M. Spouse concordance of obesity, blood pressures and serum risk factors for atherosclerosis. J Hum Hypertens 1996;10:455-9.

8. Hippisley-Cox J, Coupland C, Pringle M, Crown N, Hammersley V. Married couples' risk of same disease: cross sectional study. BMJ 2002;325:636.

9. McAdams DeMarco M, Coresh J, Woodward M, Butler KR, Kao WH, Mosley TH Jr, et al. Hypertension status, treatment, and control among spousal pairs in a middle-aged adult cohort. Am J Epidemiol 2011;174: 790-6.

10. Cheraskin E, Ringsdorf WM Jr, Setyaadmadja AT, Barrett RA, Sibley GT, Reid RW. Environmental factors in blood glucose regulation. J Am Geriatr Soc 1968;16:823-5.
11. Cobb LK, Godino JG, Selvin E, Kucharska-Newton A, Coresh J, Koton S. Spousal influence on physical activity in middle-aged and older adults: the ARIC study. Am J Epidemiol 2016;183:444-51.

12. Perry B, Ciciurkaite G, Brady CF, Garcia J. Partner influence in diet and exercise behaviors: testing behavior modeling, social control, and normative body size. PLoS One 2016;11:e0169193.

13. Ko HC. Consistency of changes in cardiovascular risk factors among spouses [master's thesis]. Seoul: Seoul National University; 1999.

14. Centers for Disease Control and Prevention (CDC). Cigarette smoking among adults: United States, 1992, and changes in the definition of current cigarette smoking. MMWR Morb Mortal Wkly Rep 1994;43: 342-6.

15. Kim JS, Oh MK, Park BK, Lee MK, Kim GJ. Screening criteria of alcoholism by alcohol use disorders identification test(AUDIT) in Korea. J Korean Acad Fam Med 1999;20:1152-9.

16. IPAQ Research Committee. Guidelines for data processing and analysis of the International Physical Activity Questionnaire (IPAQ): short and long forms. [place unknown]: International Physical Activity Questionnaire; 2005.

17. Radloff LS. The CES-D scale: a self-report depression scale for research in the general population. Appl Psychol Meas 1977;1:385-401.

18. Obesity: preventing and managing the global epidemic: report of a WHO consultation. World Health Organ Tech Rep Ser 2000;894:ixii,1-253.

19. Simonen RL, Perusse L, Rankinen T, Rice T, Rao DC, Bouchard C. Familial aggregation of physical activity levels in the Quebec Family Study. Med Sci Sports Exerc 2002;34:1137-42.

20. Morgan KJ, Zabik ME, Stampley GL. The role of breakfast in diet adequacy of the U.S. adult population. J Am Coll Nutr 1986;5:551-63.

21. Meyler D, Stimpson JP, Peek MK. Health concordance within couples: a systematic review. Soc Sci Med 2007;64:2297-310.

22. Kim HC, Kang DR, Choi KS, Nam CM, Thomas GN, Suh I. Spousal concordance of metabolic syndrome in 3141 Korean couples: a nationwide survey. Ann Epidemiol 2006;16:292-8.

23. Jee SH, Suh I, Won SY, Kim MY. Familial correlation and heritability for cardiovascular risk factors. Yonsei Med J 2002;43:160-4.

24. Goodman CR, Shippy RA. Is it contagious?: affect similarity among spouses. Aging Ment Health 2002;6:266-74.

25. Edwards B, Clarke V. The psychological impact of a cancer diagnosis on families: the influence of family functioning and patients' illness characteristics on depression and anxiety. Psychooncology 2004;13: 562-76.

26. McLeod JD. Spouse concordance for depressive disorders in a community sample. J Affect Disord 1993;27:43-52. 